\title{
VARIATIONAL DECOMPOSITION OF VECTOR FIELDS IN THE PRESENCE OF NOISE
}

\author{
Pouya Dehghani Tafti, Emrah Bostan, and Michael Unser* \\ *Biomedical Imaging Group, École Polytechnique Fédérale de Lausanne (EPFL), Switzerland
}

\begin{abstract}
We present a variational framework, and an algorithm based on the alternating method of multipliers (ADMM), for the problem of decomposing a vector field into its curl- and divergence-free components (Helmholtz decomposition) in the presence of noise. We provide experimental confirmation of the effectiveness of our approach by separating vector fields consisting of a curl-free gradient field super-imposed on a divergence-free laminar flow corrupted by noise, as well as suppressing non-zero divergence distortions in a computational fluid dynamics simulation of blood flow in the thoracic aorta. The methods developed and presented here can be used in the analysis of flow-field images and in their correction and enhancement by enforcing suitable physical constraints such as zero divergence.
\end{abstract}

Index Terms - Helmholtz decomposition, flow-field imaging, vector fields, curl, divergence, variational methods, alternating method of multipliers (ADMM)

\section{INTRODUCTION}

The emergence of new and improved modalities and techniques for in vivo measurement of flow fields and the availability of an increasing volume of medical vector-field data in recent years, motivates the consideration of practical questions related to the reconstruction, analysis, correction, and enhancement of vector field data arising in medical applications. Vector fields appear directly in medical imaging as the mathematical representation of flow and displacement, as well as indirectly due to the vectorial nature of electromagnetic and mechanical waves used in imaging. An important application of flow-field imaging in medicine is in using phase-contrast magnetic resonance imaging (PC MRI) of blood flow in the diagnosis and treatment of cardiovascular diseases, for instance by examining complex flow patterns that can develop in arteries [1].

In working with vector fields, the universal problem of decomposing them into their curl-free (irrotational) and divergence-free (solenoidal) components (Helmholtz decomposition) is of great practical importance, due to its intimate ties with the physical equations that govern flow (Navier-Stokes) and electromagnetic fields (Maxwell). Having available methods for such a decomposition is useful, for instance, in applying divergence-free corrections to measurements of incompressible blood flow. As measurement noise is always present, it is of interest to develop methods for vector field decomposition that are resilient to corruption by noise.

Continuing our previous line of work on vector field denoising and reconstruction in medical applications [2-4], in this paper

This work was partially supported by the Leenaards and Louis-Jeannet Foundations and by the European Commission under Grant ERC2010-AdG 267439-FUN-SP. we present a variational formulation and an algorithmic framework for Helmholtz decomposition of vector fields in the presence of noise. The essence of the formulation is to decompose the noisy vector field into three components: $f_{\mathrm{cf}}$ (curl-free), $f_{\mathrm{df}}$ (divergence-free), and $\boldsymbol{n}$ (noise), by minimising a suitable joint energy functional $E\left(\boldsymbol{f}_{\mathrm{cf}}, \boldsymbol{f}_{\mathrm{df}}, \boldsymbol{n}\right)$ subject to the constraint that these three components sum up to measurement vector $y$ :

$$
\begin{array}{cl}
\underset{\boldsymbol{f}_{\mathrm{cf}}, \mathrm{f}_{\mathrm{df}}, \boldsymbol{n}}{\operatorname{minimise}} & E\left(\boldsymbol{f}_{\mathrm{cf}}, \boldsymbol{f}_{\mathrm{df}}, \boldsymbol{n}\right) \\
\text { subject to } & \boldsymbol{f}_{\mathrm{cf}}+\boldsymbol{f}_{\mathrm{df}}+\boldsymbol{n}=\boldsymbol{y} .
\end{array}
$$

We refer to problems of the above type as variational decomposition. The approach we present here differs from other decomposition methods used in computational fluid dynamics (for instance those based on wavelets [5]), both in its formulation as an optimisation problem and in accounting for noise.

In the following sections we first present the general problem formulation and then an efficient numerical optimisation algorithm for its resolution, which is based on the alternating direction method of multipliers (ADMM). The effectiveness of the approach is confirmed in two experiments, before we conclude with some final remarks and directions for future work.

\section{PROBLEM FORMULATION}

We begin with a general flow reconstruction problem, where a vector field $f$ is measured by a linear system A to produce noisy measurements

$$
y=\mathrm{A} f+n
$$

( $\boldsymbol{n}$, the 'noise', is the deviation from the model). In a variational framework, we separate signal and noise by minimising an energy

$$
\alpha_{n} E_{n}(\mathbf{y}-\mathbf{A} f)+\alpha_{0} E_{0}(f)
$$

where $E_{n}(y-\mathbf{A} f)$ is the so-called fidelity term and $E_{0}(f)$ is the regulariser, which corresponds to our (probabilistic or nonprobabilistic) prior on $f$, and where $\alpha_{0}, \alpha_{n}$ are parameters determining the contribution of each term to the total energy (only the ratio between the two is important). In view of (1), we may symmetrise the place of signal and noise, by formulating our denoising problem as a variational decomposition of $\boldsymbol{y}$ :

$$
\begin{array}{ll}
\underset{\boldsymbol{f}, \boldsymbol{n}}{\operatorname{minimise}} & \alpha_{0} E_{0}(\boldsymbol{f})+\alpha_{n} E_{n}(\boldsymbol{n}) \\
\text { subject to } & \mathbf{A} \boldsymbol{f}+\boldsymbol{n}=\boldsymbol{y} .
\end{array}
$$

The additive combination of energies is consistent with the assumption that the signal and noise are independent. In a discrete regime, the simplest measurement operator is the identity and the corresponding decomposition is $\boldsymbol{y}=\boldsymbol{f}+\boldsymbol{n}$. This shall be the case for the rest of this paper. 
If, in addition to denoising, we consider the decomposition of $f$ into curl- and divergence-free components $f_{\text {cf }}$ and $f_{\text {df }}$ respectively, we shall have the decomposition

$$
y=f_{\mathrm{cf}}+f_{\mathrm{df}}+n .
$$

By definition, the curl- and divergence-free components are subject to the constraints curl $f_{\mathrm{cf}}=0, \operatorname{div} f_{\mathrm{df}}=0$, in regions where the field is differentiable.

In practice, we work with discretised fields as well as discretised curl and divergence operators, which, in the simplest case, are based on finite differences [3, 4]. Across field discontinuities (fluid interfaces, boundaries, etc.), these operators assign non-zero values to the discretised $\operatorname{curl}_{d} f_{\mathrm{cf}}$ and $\operatorname{div}_{d} f_{\mathrm{df}}$ (subscript $d$ stands for discrete). Moreover, in other smoother areas, the previous terms may have small non-zero amplitudes due to the approximation of continuous operators. It is therefore useful to relax the above constraints as

$$
E_{\mathrm{cf}}\left(\operatorname{curl}_{d} f_{\mathrm{cf}}\right) \leq \varsigma_{\mathrm{cf}}, \quad E_{\mathrm{df}}\left(\operatorname{div}_{d} f_{\mathrm{df}}\right) \leq \varsigma_{\mathrm{df}},
$$

where $E_{\mathrm{cf}}$ and $E_{\mathrm{df}}$ are energy functionals (potentials) corresponding to our variational prior on the distribution of the energy of $\operatorname{curl}_{d} f_{\mathrm{cf}}$ and $\operatorname{div}_{d} f_{\mathrm{df}}$, and where $\varsigma_{\mathrm{cf}}$ and $\varsigma_{\mathrm{df}}$ are the respective energies of the said quantities. A similar energy principle is applied to the noise (modelling error):

$$
E_{n}(\boldsymbol{n}) \leq \varsigma_{n} .
$$

The noise energy functional $E_{n}$ is typically defined in proportion to the sample variance:

$$
E_{n}(\boldsymbol{n})=\frac{1}{2}\|\boldsymbol{n}\|_{2}^{2}=\frac{1}{2} \sum_{\boldsymbol{k} \in \mathscr{K}}|\boldsymbol{n}[\boldsymbol{k}]|^{2},
$$

conforming to a Gaussian prior if we wish to make a probabilistic interpretation of the above ( $\mathscr{K}$ is the set of sample indices). In practice, the parameters $\varsigma_{\mathrm{cf}}$, $\varsigma_{\mathrm{df}}$, and $\varsigma_{n}$ are a priori unknown and are left as tunable parameters of the method with the possibility of adapting them to each problem.

As noted before, our prior on $f$ is represented by a potential $E_{0}(f)$. As our solution, we seek the vector field that minimises $E_{0}$ subject to the above constraints:

$$
\begin{array}{cl}
\underset{f_{\mathrm{cf}}, f_{\mathrm{df}}, \boldsymbol{n}}{\operatorname{minimise}} & \alpha_{0} E_{0}\left(f_{\mathrm{cf}}+f_{\mathrm{df}}\right) \\
\text { subject to } & E_{\mathrm{cf}}\left(\operatorname{curl}_{d} f_{\mathrm{cf}}\right) \leq \varsigma_{\mathrm{cf}}, E_{\mathrm{df}}\left(\operatorname{div}_{d} f_{\mathrm{df}}\right) \leq \varsigma_{\mathrm{df}}, E_{n}(\boldsymbol{n}) \leq \varsigma_{n}, \\
& \boldsymbol{f}_{\mathrm{cf}}+\boldsymbol{f}_{\mathrm{df}}+\boldsymbol{n}=\boldsymbol{y} .
\end{array}
$$

The Lagrange relaxation of the above problem for the first three constraints yields:

$$
\begin{aligned}
\underset{f_{\mathrm{cf}}, f_{\mathrm{df}}, \boldsymbol{n}}{\operatorname{minimise}} \quad \alpha_{0} E_{0}\left(f_{\mathrm{cf}}+f_{\mathrm{df}}\right) & +\alpha_{\mathrm{cf}} E_{\mathrm{cf}}\left(\operatorname{curl}_{d} f_{\mathrm{cf}}\right) \\
& +\alpha_{\mathrm{df}} E_{\mathrm{df}}\left(\operatorname{div}_{d} f_{\mathrm{df}}\right)+\alpha_{n} E_{n}(\boldsymbol{n})
\end{aligned}
$$

subject to $f_{\text {cf }}+f_{\mathrm{df}}+n=y$,

where we identify the fidelity and regularisation terms of (2) in addition to two new terms for Helmholtz decomposition. The $\alpha$ parameters in the Lagrange formulation take the place of $\varsigma_{\mathrm{cf}}, \varsigma_{\mathrm{df}}, \varsigma_{n} \mathrm{~s}$ as algorithm parameters.

To make the above general formulation specific, in the present work we use the quadratic energy defined in (3) for the noise component; for $E_{\mathrm{cf}}$ and $E_{\mathrm{df}}$ we use the $\ell_{1}$ norm to promote sparsity, having in mind that ideally curl $_{d} f_{\text {cf }}$ and curl $_{d} f_{\text {df }}$ should be concentrated mostly at field discontinuities (which happen across lower dimensional manifolds and are therefore sparse); and, finally, we choose $E_{0}$ to be of the form

$$
E_{0}(\boldsymbol{f})=\frac{1}{p}\|\mathrm{R} \boldsymbol{f}\|_{p}^{p}
$$

with $p=1$ or 2 depending on the characteristics of the underlying field, where $\mathrm{R}$ is a differential operator that is meant to capture the dependency structure of $f$ in space and possibly in time. The $\ell_{p}$ norms here are defined over the finite set $\mathscr{K}$ of sample indices:

$$
\|x\|_{p}^{p}=\sum_{k \in K}\left|x_{k}\right|^{p}
$$

where $|\cdot|$ denotes the absolute value of a scalar or the magnitude of a vector as appropriate (for a discussion of vector norms see [4]).

The final energy to minimise, subject to $f_{\mathrm{cf}}+f_{\mathrm{df}}+\boldsymbol{n}=\boldsymbol{y}$, is thus of the form

$$
\begin{aligned}
E\left(f_{\mathrm{cf}}, f_{\mathrm{df}}, \boldsymbol{n}\right)=\frac{\alpha_{0}}{p}\left\|\mathrm{R}\left(f_{\mathrm{cf}}+f_{\mathrm{df}}\right)\right\|_{p}^{p} & +\alpha_{\mathrm{cf}}\left\|\operatorname{curl}_{d} f_{\mathrm{cf}}\right\|_{1} \\
& +\alpha_{\mathrm{df}}\left\|\operatorname{div}_{d} f_{\mathrm{df}}\right\|_{1}+\frac{\alpha_{n}}{2}\|\boldsymbol{n}\|_{2}^{2} .
\end{aligned}
$$

\section{SOLUTION VIA CONVEX OPTIMISATION}

We transform our original optimisation problem (4) with the energies defined in (6), to the following equivalent form, which-as we shall see-is amenable to resolution by the alternating direction method of multipliers (ADMM):

$$
\begin{aligned}
\underset{f_{\mathrm{cf}}, f_{\mathrm{df}}, \boldsymbol{u}, \boldsymbol{v}, \boldsymbol{w}}{\operatorname{minimise}} & \frac{\alpha_{0}}{p}\|\boldsymbol{u}\|_{p}^{p}+\alpha_{\mathrm{cf}}\|\boldsymbol{v}\|_{1}+\alpha_{\mathrm{df}}\|w\|_{1}+\frac{\alpha_{n}}{2}\left\|\boldsymbol{f}_{\mathrm{cf}}+\boldsymbol{f}_{\mathrm{df}}-\boldsymbol{y}\right\|_{2}^{2} \\
\text { subject to } & \boldsymbol{u}=\mathrm{R}\left(\boldsymbol{f}_{\mathrm{cf}}+\boldsymbol{f}_{\mathrm{df}}\right), \quad \boldsymbol{v}=\operatorname{curl}_{d} \boldsymbol{f}_{\mathrm{cf}}, \quad w=\operatorname{div}_{d} \boldsymbol{f}_{\mathrm{df}} .
\end{aligned}
$$

This formulation leads to the (scaled) augmented Lagrangian [6]

$$
\begin{aligned}
L\left(\boldsymbol{f}_{\mathrm{cf}}, \boldsymbol{f}_{\mathrm{df}},\right. & \boldsymbol{u}, \boldsymbol{v}, w, \boldsymbol{\gamma}, \boldsymbol{\delta}, \boldsymbol{\epsilon}) \\
= & \frac{\alpha_{0}}{p}\|\boldsymbol{u}\|_{p}^{p}+\alpha_{\mathrm{cf}}\|\boldsymbol{v}\|_{1}+\alpha_{\mathrm{df}}\|w\|_{1}+\frac{\alpha_{n}}{2}\left\|\boldsymbol{f}_{\mathrm{cf}}+\boldsymbol{f}_{\mathrm{df}}-\boldsymbol{y}\right\|_{2}^{2} \\
& +\frac{\rho_{0}}{2}\left\|\boldsymbol{u}-\mathrm{R}\left(\boldsymbol{f}_{\mathrm{cf}}+\boldsymbol{f}_{\mathrm{df}}\right)\right\|_{2}^{2}+\rho_{0}\left\langle\boldsymbol{\gamma}, \boldsymbol{u}-\mathrm{R}\left(\boldsymbol{f}_{\mathrm{cf}}+\boldsymbol{f}_{\mathrm{df}}\right)\right\rangle \\
& +\frac{\rho_{\mathrm{cf}}}{2}\left\|\boldsymbol{v}-\operatorname{curl}_{d} \boldsymbol{f}_{\mathrm{cf}}\right\|_{2}^{2}+\rho_{\mathrm{cf}}\left\langle\boldsymbol{\delta}, \boldsymbol{v}-\operatorname{curl}_{d} \boldsymbol{f}_{\mathrm{cf}}\right\rangle \\
& +\frac{\rho_{\mathrm{df}}}{2}\left\|w-\operatorname{div}_{d} \boldsymbol{f}_{\mathrm{df}}\right\|_{2}^{2}+\rho_{\mathrm{df}}\left\langle\epsilon, w-\operatorname{div}_{d} \boldsymbol{f}_{\mathrm{df}}\right\rangle
\end{aligned}
$$

with scaled Lagrange multipliers $\gamma, \delta$, and $\epsilon$. The augmented Lagrangian is to be simultanesouly minimised in $f_{\mathrm{cf}}, f_{\mathrm{df}}, \boldsymbol{u}, \boldsymbol{v}$, and $w$, and maximised in $\boldsymbol{\gamma}, \boldsymbol{\delta}$, and $\epsilon$ (the $\rho$ s are arbitrary positive parameters). We use ADMM for this optimisation [6]. For the above problem, each ADMM iteration involves minimising $L$ sequentially in each of the first five variables, and taking gradient steps in the direction of each of the last three.

For $f_{\mathrm{cf}}$ and $f_{\mathrm{df}}$ we find the minimiser in each step by setting the partial derivatives with respect to each of them equal to zero and solving the resulting linear systems. This leads to the update equations (superscript $*$ denotes the adjoint operator):

$$
\begin{aligned}
f_{\mathrm{cf}}= & \left(\alpha_{n}+\rho_{0} \mathrm{R}^{*} \mathrm{R}+\rho_{\mathrm{cf}} \operatorname{curl}_{d}^{*} \operatorname{curl}_{d}\right)^{-1} \\
& \left(\alpha_{n}\left(\boldsymbol{y}-f_{\mathrm{df}}\right)+\rho_{0} \mathrm{R}^{*}\left(\boldsymbol{u}-\mathrm{R} f_{\mathrm{df}}+\boldsymbol{\gamma}\right)+\rho_{\mathrm{cf}} \operatorname{curl}_{d}^{*}(\boldsymbol{v}+\boldsymbol{\delta})\right), \\
f_{\mathrm{df}}= & \left(\alpha_{n}+\rho_{0} \mathrm{R}^{*} \mathrm{R}+\rho_{\mathrm{df}} \operatorname{div}_{d}^{*} \operatorname{div}_{d}\right)^{-1} \\
& \left(\alpha_{n}\left(\boldsymbol{y}-f_{\mathrm{cf}}\right)+\rho_{0} \mathrm{R}^{*}\left(\boldsymbol{u}-\mathrm{R} f_{\mathrm{cf}}+\boldsymbol{\gamma}\right)+\rho_{\mathrm{df}} \operatorname{div}_{d}^{*}(w+\epsilon)\right) .
\end{aligned}
$$


Regarding as to whether $p=1$ or 2 is used for the $\boldsymbol{u}$ term, the minimisation step in $u$ is determined as follows. For $p=2$, we set the partial derivative with respect to $\boldsymbol{u}$ to zero in order to find the update equation

$$
\boldsymbol{u}=\frac{\rho}{\alpha_{0}+\rho}\left(\mathrm{R}\left(\boldsymbol{f}_{\mathrm{cf}}+f_{\mathrm{df}}\right)-\boldsymbol{\gamma}\right) .
$$

For $p=1$ the minimiser is obtained by expanding the $\ell_{1}$ and squared $\ell_{2}$ norms as sums and minimising them term by term. The solution is given by vector shrinkage:

$$
\boldsymbol{u}=\left(1-\frac{1}{\frac{\rho_{0}}{\alpha_{0}}\left|\mathrm{R}\left(f_{\mathrm{cf}}+f_{\mathrm{df}}\right)-\gamma\right|}\right)_{+}\left(\mathrm{R}\left(f_{\mathrm{cf}}+f_{\mathrm{df}}\right)-\gamma\right),
$$

where $(a)_{+}=a$ is the positive part of $a$ and $|\cdot|$ denotes the magnitude of a vector (or tensor, depending on the range of $\mathrm{R}$ ).

The updates for $v$ and $w$ take a similar form:

$$
\begin{aligned}
& \boldsymbol{v}=\left(1-\frac{1}{\frac{\rho_{\mathrm{cf}}}{\alpha_{\mathrm{cf}}}\left|\operatorname{curl}_{d} f_{\mathrm{cf}}-\delta\right|}\right)+\left(\operatorname{curl}_{d} f_{\mathrm{cf}}-\delta\right), \\
& w=\left(1-\frac{1}{\frac{\rho_{\mathrm{df}}}{\alpha_{\mathrm{df}}}\left|\operatorname{div}_{d} f_{\mathrm{df}}-\epsilon\right|}\right)+\left(\operatorname{div}_{d} f_{\mathrm{df}}-\epsilon\right) .
\end{aligned}
$$

Finally, for $\boldsymbol{\gamma}, \boldsymbol{\delta}$, and $\epsilon$ the update equations are given by simple gradient ascent steps:

$$
\begin{aligned}
& \boldsymbol{\gamma}=\boldsymbol{\gamma}_{\text {prev. }}+\boldsymbol{u}-\mathrm{R}\left(f_{\mathrm{cf}}+f_{\mathrm{df}}\right) \\
& \boldsymbol{\delta}=\boldsymbol{\delta}_{\text {prev. }}+\boldsymbol{v}-\operatorname{curl}_{d} f_{\mathrm{cf}} \\
& \epsilon=\epsilon_{\text {prev. }}+\boldsymbol{w}-\operatorname{div}_{d} f_{\mathrm{df}} .
\end{aligned}
$$

The final ADMM iteration consists of updates (A1)-(A8) applied in sequence (with either (A3) or (A3') depending on $p$ ).

\section{EXPERIMENTS}

In our first experiment, the goal was to separate a 3D gradient field (curl-free), from an incompressible laminar flow (divergence-free), in the presence of additive white Gaussian noise (total signal to noise ratio (SNR) of $10 \mathrm{~dB}$ ). The curl-free field in question was the gradient of the function $\phi(x, y, z)=x y \exp \left(-x^{2}-y^{2}-z^{2}\right)$ over the region $[-2,2] \times[-2,2] \times[-2,2]$ with sampling step 0.1 . For the incompressible component we simulated a laminar flow with a typical parabolic intensity profile inside a cylindrical pipe of radius 1 oriented along the $z$ axis.

The following parameters were used in the experiment. For regularisation operator $\mathrm{R}$, we chose the simplest first order operator, namely the vector gradient. $E_{0}$ was defined with $p=1$ (update equation (A3')). Since the curl of $f_{\mathrm{cf}}$ and the divergence of $f_{\text {df }}$ (and thus the corresponding terms in (6)) are expected nearly to vanish, the values of parameters $\alpha_{\mathrm{cf}}$ and $\alpha_{\mathrm{df}}$ do not considerably affect the final outcome. They can both be chosen to be very large (we set them to $10^{6}$ ). $\alpha_{0}$ can always be set to 1 without loss of generality (equivalent to dividing the objective by $\alpha_{0}$ ). Finally, $\alpha_{n}$ can be tuned according to noise level for optimal denoising, or set using a method such as generalised cross-validation or SURE [7]. However, here we simply chose a value of 5 for it for the purpose of demonstrating the decomposition without demanding optimal denoising performance. To solve linear systems (A1) and (A2) we used the conjugate gradient method with a maximum of 500 iterations per ADMM step and a tolerance of $10^{-12}$.
Table 1. Input and output SINR for curl- and divergence-free components in the first experiment (see text); all values in $\mathrm{dB}$

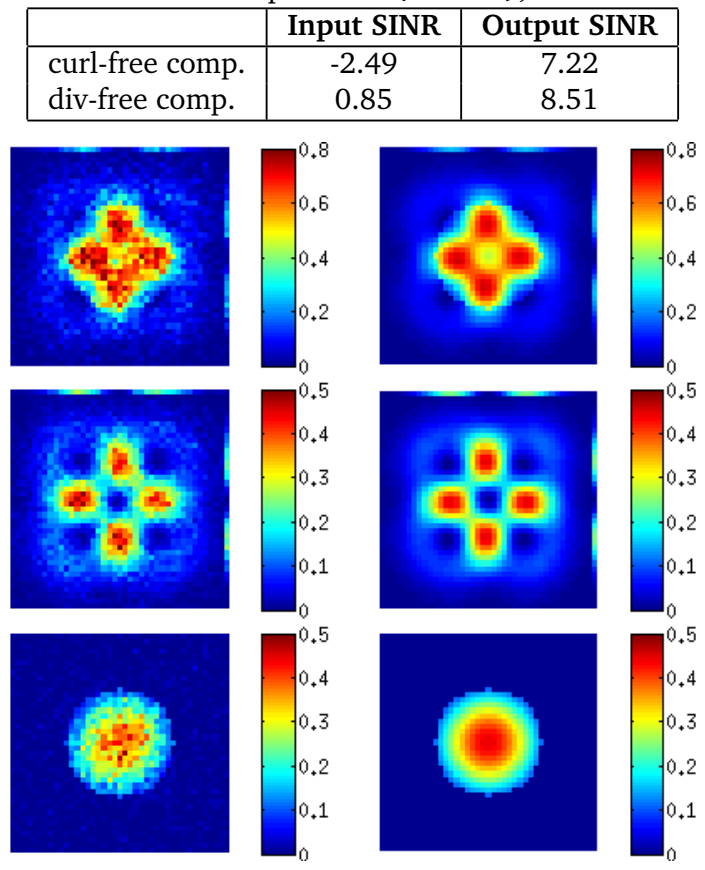

Fig. 1. Amplitude $z$ cross-sections for the decomposition of an analytical vector field into curl- and div-free components (first experiment; see text). Top left: input (sum of curl- and div-free fields corrupted by noise); top right: original field; centre left: recovered curl-free component; centre right: original curl-free component; bottom left: recovered div-free component; bottom right: original div-free component.

The initial signal to interference plus noise ratio (SINR) for the two components and the same quantity after ADMM iterations are given in Table 1. Figure 1 shows the original analytical components and the noisy measurements together with the recovered field components. A very good separation is visibly achieved after as few as $5 \mathrm{ADMM}$ iterations, in spite of the significant overlap between the large-intensity regions of the two components.

In the second experiment, we aimed to suppress distortion appearing as a compressible term in a computational phantom of blood flow in the thoracic aorta. The simplified aorta geometry used in this simulation was extracted from a 3D, ECG-gated, whole-heart, balanced Steady-State Free Precession (BSSFP) MRI dataset acquired on a 3T clinical scanner (Siemens AG, Erlangen, Germany) with a 3D radial trajectory based on a spiral phyllotaxis pattern [8]. The flow, which was assumed to be laminar, was computed by numerically solving the Navier-Stokes equations (using the transient solver of ANSYS ${ }^{\circledR} \mathrm{CFX}^{\circledR}$ to account for the time-dependent inflow), modelling blood as an incompressible Newtonian fluid with a density of $1040 \mathrm{Kg} / \mathrm{m}^{3}$ and a dynamic viscosity of $0.004 \mathrm{~Pa}$.s., and ascribing no-slip conditions to the walls of the thoracic aorta.

The flow was then distorted by the addition of a gradient field and the outcome was further degraded by independent additive white Gaussian noise (10 dB degradation relative to the already distorted field), to reach a final SINR of $-0.55 \mathrm{~dB}$ for the flow. Since the flow is incompressible (divergence-free), it can be separated from the gradient distortion using the proposed method. 
Table 2. Input and output SINR for incompressible blood flow in the second experiment (see text); all values in $\mathrm{dB}$

\begin{tabular}{|c|c|c|}
\hline Input SINR & Output SINR & Output SINR (thresholded) \\
\hline-0.55 & 4.41 & 11.00 \\
\hline
\end{tabular}

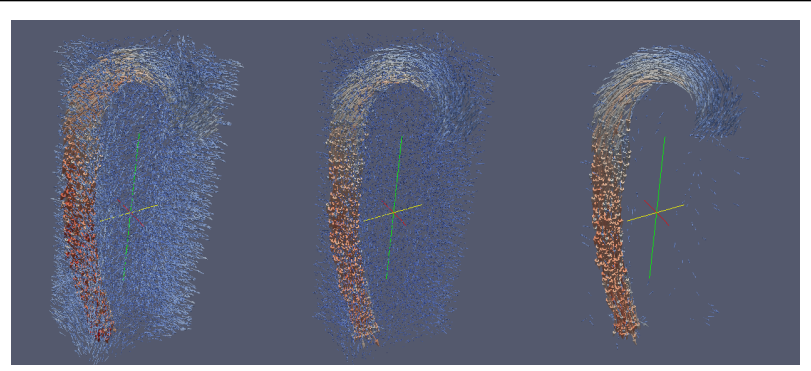

Fig. 2. Suppression of non-zero-divergence distortion and noise for blood flow in the thoracic aorta, vector glyph visualisation (second experiment; see text). Left: distorted field; centre: divergence-free output; right: divergence-free output after thresholding.

The algorithm parameters were set as follows. For the regularisation energy $\left(E_{0}\right)$, we used a quadratic term $(p=2)$ with the vector gradient operator. $\alpha_{\mathrm{cf}}, \alpha_{\mathrm{df}}$, and $\alpha_{0}$ were assigned the same values as in the previous experiment. For faster execution, we reduced the number of conjugate gradient iterations in steps (A1) and (A2) to 100. Since reducing solver iterations in this case is qualitatively comparable to additional regularisation, we increased $\alpha_{n}$ to 100 to compensate for this (once again without optimising the value for best performance).

A total of $10 \mathrm{ADMM}$ iterations were needed to achieve the separation performance reported in Table 2 . If we follow the decomposition by a simple magnitude thresholding to suppress the attenuated remnants of the distortive field, we achieve a significant further improvement (thresholding is not possible before decomposition since the two components have comparable magnitudes). Vector glyph visualisations of the distorted and corrected flow, produced using ParaView visualisation software (KITWare Inc, NY, USA), can be seen in Figure 2. Figure 3 shows streamline visualisations of the same two fields, produced using EnSight (CEI, NC, USA). In addition to suppressing the non-zero-divergence distortion in the flow, in terms of noise reduction, for the sum of the two terms we achieved an SNR improvement from $10 \mathrm{~dB}$ to $10.97 \mathrm{~dB}$, which is comparable with the state of the art [2, 3]. Note that all these results were obtained without any substantial optimisation of algorithm parameters.

\section{CONCLUSION}

In this paper we presented a variational scheme for the decomposition of vector fields into their curl- and divergence-free components (Helmholtz decomposition) in the presence of noise. We formulated the decomposition as a convex optimisation problem and proposed a numerical algorithm based on the alternating direction method of multipliers (ADMM) applied to the augmented Lagrangian for its resolution. The effectiveness of the approach was demonstrated by experiments consisting in the separation of analytically computed curl- and divergence-free components in the presence of noise (first experiment), and the suppression of non-zero-divergence distortion and additive noise in simulated incompressible thoracic blood flow (second experiment). Possibilities for future research include using temporal regularisation for

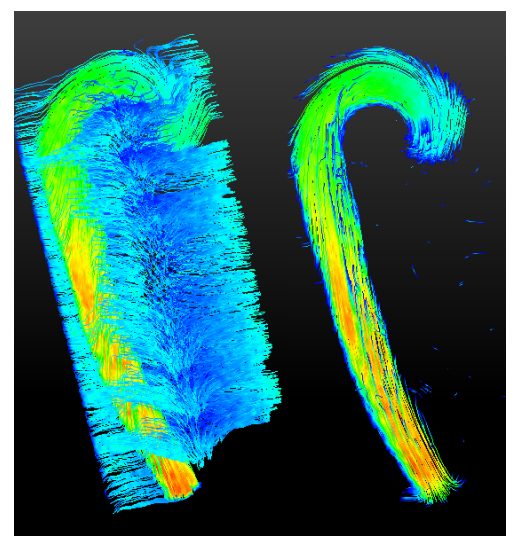

Fig. 3. Suppression of non-zero-divergence distortion and noise in blood flow in the thoracic aorta, streamline visualisation (second experiment; see text). Left: distorted field; right: recovered divergence-free field.

the global Helmholtz decomposition of 4D vector field data, and adapting the approach to solve other problems of interest such as phase-unwrapping in phase-contrast MR imaging of flow fields.

\section{ACKNOWLEDGMENTS}

The authors thank Orestis Vardoulis and Prof. Nikolaos Stergiopulos from the Laboratory of Hemodynamics and Cardiovascular Technology, EPFL, Switzerland, for kindly providing the blood flow data used in the second experiment.

\section{REFERENCES}

[1] A. Frydrychowicz, R. Arnold, D. Hirtler, C. Schlensak, A. F. Stalder, J. Hennig, M. Langer, and M. Markl, "Multidirectional flow analysis by cardiovascular magnetic resonance in aneurysm development following repair of aortic coarctation," J. Cardiovasc. Magn. Reson., vol. 10, no. 1, p. 30, 2008.

[2] E. Bostan, P. D. Tafti, and M. Unser, "A dual algorithm for $\mathrm{L}_{1}$ regularized reconstruction of vector fields," in Proc. 9th IEEE Int. Symp. Biomed. Imaging (ISBI'12), 2012, pp. 1579-1582.

[3] P. D. Tafti, R. Delgado-Gonzalo, A. F. Stalder, and M. Unser, "Variational enhancement and denoising of flow field images," in Proc. 8th IEEE Int. Symp. Biomed. Imaging (ISBI'11), 2011, pp. 1061-1064.

[4] P. D. Tafti and M. Unser, "On regularized reconstruction of vector fields," IEEE Trans. Image Process., vol. 20, no. 11, pp. 3163-3178, 2011.

[5] E. Deriaz, M. Farge, and K. Schneider, "Craya decomposition using compactly supported biorthogonal wavelets," Appl. Comput. Harmon. Anal., vol. 28, no. 4, pp. 267-284, 2010.

[6] S. Boyd, N. Parikh, E. Chu, B. Peleato, and J. Eckstein, "Distributed optimization and statistical learning via the alternating direction method of multipliers," Found. Trends Mach. Learn., vol. 3, no. 1, pp. 1-122, 2011.

[7] S. Ramani, T. Blu, and M. Unser, "Monte-Carlo SURE: A blackbox optimization of regularization parameters for general denoising algorithms," IEEE Trans. Image Process., vol. 17, no. 9, pp. 1540-1554, 2008.

[8] D. Piccini, A. Littmann, S. N.-Vallespin, and O. M. Zenge, "Spiral phyllotaxis: The natural way to construct a 3D radial trajectory in MRI," Magn. Reson. Med., vol. 66, no. 4, pp. 1049-1056, 2011. 